so mehr Manna kann gewonnen werden. Ein schmaler Streif des Stammes muss immer unbeschädigt, glatt bleiben, damit die Manna leichter herabrinnen kann; ist der Baum auf den übrigen Seiten von unten nach oben ganz geschnitten, dann : benutzt man im nächsten Jahre die Stämme zwischen den alten vernarbten Wunden. Canellata wird von den oberen Einschnitten, deren ich bis $\mathbf{4 0}$ an manchen Bäumen zählte, gewonnen; denn weiter aufwärts ist der Saft nicht mehr so fett, trocknet leichter und es bilden sich daher die Röbren und die flachen Stücke. Wird die Manna von den Bäumen genommen, so ist sie noch nicht trocken genug, sie muss auf Brettern ausgebreitet, vollständig getrocknet werden, und wird dann erst in Kisten gelegt. Was beim Abnehmen der Canoli am Baume hängen bleibt, wird heruntergeschabt und dieses bildet die Manna canellata in fragmentis. Anhaltend trocknes Wetter während der Zeit der Einschnitte ist also unerlässliche Bedingung, um viele und schöne Manna zu gewinnen. Wenn jedoch der Baum zu sehr in den Samen schiesst, so wird trotz der günstigsten Witterung nur wenig Manna erzielt. Canellata, Canellata in fragment. und Capace, wohl ebenso die Abstufung bei Gerace, kommen demnach gleichzeitig von einem Stamme. Je jünger der Baum ist, desto mehr wird Canellata, und je älter er ist, desto mehr wird Capace oder Gerace gewonnen, die in Sicilien als in sorte bezeichnet wird und vielleicht durch ärztliche Versuche als die wirksamste der Mannasorten erkannt werden dürfte.

\title{
Kurze Notizen über Radix Sambul und Radix Rhel.
}

(Aus einem Briefe des Apothekers Pfeffer in St. Petersburg an Dr. Bley.)

Die Sambul-Wurzel wird bei uns als Infusum und als Tinctur in vielen Fällen als ein ausgezeichnetes neues Heilmittel angewendet.

Ein Reisender hat mir eine Kiste ungeschälter Rha- 
barber aus China mitgebracht. Da habe ich nun gesehen, dass durchaus in jedem Stuicke ohne Ausnahme ein Loch war, was also bestimmt darthut, dass die frischen Wurzeln durchbohrt, auf Fäden aufgezogen und getrocknet werden. Nachher beim Abschälen und Reinigen werden diese Löcher ausgebohrt oder ausgeschnitten, weil sie voll von Würmern sind. Beim Oeffnen der Kiste flogen mir Hunderte von Motten entgegen; in vielen Stücken, welche ich abgefeilt und gereinigt habe, fand ich noch Würmer.

Von beiden Wurzeln übersende ich einige Exemplare anbei Ihnen zu, so wie ein Exemplar des Statuts unserer pharmaceutischen Wittwen - und Waisen-Casse.

Zusalz. Dr. Thielmann hat nach einem Berichte im medicinischen Journale Russlands, Jahrgang 1847, Heft I, über die Rad. Sambul Mittheilungen gemacht, woraus hier einige Notizen folgen.

Diese Wurzel wurde zuerst vor elf Jahren durch einen persischen Kaufmann nach Moskau gebracht, wo sie Anfangs ihres starken Moschusgeruchs wegen an Parfumeurs verkauft wurde. Es scheint ohne $Z$ weifel, dass selbige von einer Umbellifere abstamme und der Angelica nahe verwandt sei. Dr. Thielmann hat selbige bei melhr als 200 Kranken versucht und gefunden, dass dieselbe

1) als Infusum und Tinctur den Verdauungsprocess befördere.

2) In zwei Fällen von Chorea Sti Viti bewirkte die Wurzel sowobl bei einem 15 jährigen Mädchen, als einem 49jährigen Jüngling vollkommene Heilung.

3) Bei Anästhesie der Blasennerven wirkte selbige ausgezeichnet. Es dürften überhaupt die chronischen Nervenkrankheiten einen grossen Wirkungskreis für die Wirkung der Rad. Sambul darbieten.

b) Gegen Durchfälle scheint diese Wurzel die meisten bisher bekannten Mittel an Wirksamkeit zu ubertreffen.

5) Bei Cholera sporadica wurde selbige bei 5 Individuen als Infusum oder Infuso-Decoctum mit dem ginstigsten Erfolge angewendet.

b. 